\title{
Efficacy of arthroscopic loose body removal for knee osteoarthritis
}

\author{
BAOXIANG ZHAO ${ }^{*}$, YIBO YU ${ }^{2,3^{*}}$, WENQUAN LIU ${ }^{3}$ and JIAN DU ${ }^{4}$ \\ ${ }^{1}$ Department of Orthopaedics, Linyi People's Hospital, Linyi, Shandong 276000; \\ ${ }^{2}$ Department of Orthopaedics, Qilu Hospital of Shandong University, Jinan, Shandong 250012; \\ ${ }^{3}$ Department of Orthopaedics, Chinese Medicine Hospital in Linyi City, Linyi, Shandong 276002; \\ ${ }^{4}$ Department of Orthopaedics, Lanshan District People's Hospital, Linyi, Shandong 276000, P.R. China
}

Received July 14, 2016; Accepted April 28, 2017

DOI: $10.3892 /$ etm.2017.5564

\begin{abstract}
The purpose of the present study was to explore the efficacy of arthroscopic loose body removal for knee osteoarthritis (KOA). A total of 23 patients with KOA were enrolled and randomly received conservative treatment (conservative group; $n=10$ ) or loose body removal surgery (surgery group; $\mathrm{n}=13$ ). The serum levels of disease activity indices, including hypersensitive C-reactive protein (hs-CRP), erythrocyte sedimentation rate (ESR) and synovial inflammatory factors [interleukin (IL)-1 and IL-6] were detected prior to surgery, and at 4 days, 2 or 4 weeks after surgery. All patients were followed up for 2 years and the cure rate was estimated. No significant difference was identified in pre-operative plasma levels of hs-CRP and ESR as well as the synovial concentration of IL- 1 and IL- 6 between the two groups (all P>0.05). At 2 and 4 weeks after treatment, the levels of these parameters in the surgery group were significantly lower than those in the conservative group (all $\mathrm{P}<0.05$ ), although the maximum value of these parameters was higher in the surgery group than in the conservative group at 4 days after surgery. The cure rate for KOA in the surgery group was significantly higher than that in the conservative group. In conclusion, the results demonstrated that arthroscopic loose body removal is a more effective treatment than conservative therapy for KOA.
\end{abstract}

\section{Introduction}

Osteoarthritis (OA) is a joint disease characterized by articular cartilage fibrosis, derangement, ulceration and loss caused by a variety of factors (1). OA primarily occurs in weight-bearing joints, including knees, hips, ankles, hands and spine

Correspondence to: Professor Jian Du, Department of Orthopaedics, Lanshan District People's Hospital, 15 Kaopeng Street, Linyi, Shandong 276000, P.R. China

E-mail: xnv333@126.com

\section{*Contributed equally}

Key words: arthroscopy, loose body removal, knee osteoarthritis, therapy joints, and is a major burden on individuals and social care systems (2). Knee osteoarthritis (KOA) is a disease characterized by the degeneration of articular cartilage, leading to subchondral bone proliferation, cartilage exfoliation and the gradual destruction and dysfunction of the knee joint (3-6). The clinical features of this disorder include joint pain, movement limitation, poor muscle strength and stability, and walking impairment, which severely affect the quality of life of affected individuals (7).

At present, the main therapeutic options for KOA are conservative or surgical treatment (8). The conservative treatment includes drug treatment and non-drug therapy. The first-line drugs, including non-steroidal anti-inflammatory drugs (NSAIDs) and acetaminophen, effectively alleviate the symptoms of osteoarthritis (9). Furthermore, intra-articular injection of corticosteroids and hyaluronic acid has a significant effect on the remission of symptoms, reduction of joint effusion and protection of cartilage (10). However, the long-term use of these drugs inevitably leads to gastrointestinal side effects or other severe adverse outcomes (11).

Alternatively, patients with severe KOA may be treated by surgeries, including arthroscopic removal of loose body, joint debridement, osteotomy, arthrodesis and arthroplasty (artificial joint replacement) (12). Intra-articular loose bodies, which are formed due to articular cartilage atrophy, degeneration and necrosis after cartilage malnutrition or developmental disorders, are chondral, osseous or osteochondral fragments located in the articular cavity (13). Loose bodies that move freely in the joint cavity may lead to joint pain, locking and swelling (12). According to their structure, intra-articular loose bodies may be divided into fibrous, cartilaginous bony, cartilaginous, bony and crystalline loose bodies or exogenous articular foreign matter (14). The most common type is bony loose bodies, which have a core of bone structure covering the outer periphery of the cartilage, or cartilaginous loose bodies with a round shape and smooth surface, which are entirely composed of cartilage (15). Smaller loose bodies tend to move easier in the joint cavity, causing joint pain and locking in unpredictable positions (16). Removal of loose bodies not only relieves long-term suffering of patients, but also restores joint function and the ability to live independently (17). The aim of the present study was to compare the therapeutic efficacy of conservative treatment and arthroscopic loose body removal for KOA. 


\section{Materials and methods}

Patients. A total of 23 patients with KOA were eligible for enrollment in the present study. KOA was diagnosed according to the clinical and radiographic criteria of the American College of Rheumatology from 1995, which were as follows: i) Knee pain persisting for $\geq 14$ days within one month prior to treatment; ii) bony crepitus occurred during activity of knee joints; ii) X-ray demonstrated osteophyte formation in knee joints; iv) swelling of knee joint; v) morning stiffness of the knee joint for $\leq 30 \mathrm{~min}$; and vi) Patient age $>50$ years. Patients fulfilling the criteria $\mathrm{i}$ and iii or $\mathrm{i}$, ii, iv and $\mathrm{v}$ may be diagnosed with KOA. Prior written informed consent was obtained from each patient and the study was approved by the ethics review board of Linyi People's Hospital (Linyi, China).

Patient grouping and therapy. Based on the balance of indications and the wishes of the patients or their family members, 23 patients received conservative treatment (conservative group; $\mathrm{n}=10$ ) or loose body removal surgery (surgery group; $\mathrm{n}=13$ ). In the conservative group, the indications were as follows: Symptoms of knee pain, swelling and joint dysfunction; joint space narrowing without loose body formation as demonstrated by X-ray. Intra-articular injection of NSAIDs (acetaminophen, $<4,000 \mathrm{mg}$ per day) was administered to these patients.

In the surgery group, loose body removal surgery was performed in patients who had the following indications: Age, 40-50 years; normal blood coagulation; no history of hemophilia, anemia, malnutrition or severe disorders of heart, lung, liver or kidney; knee joint motions were severely affected but without fluctuant swelling; and X-ray demonstrated the formation of loose bodies in the knee. First, patients with loose bodies above the patella $(n=3)$ received local anesthesia, while epidural anesthesia was given to patients with loose bodies in the rear side of the knee $(n=5)$ and the knee space $(\mathrm{n}=5)$. Minimally invasive techniques were then performed to remove the loose bodies, using an S-shaped incision for loose bodies in the rear side of the knee and an oblique incision for those in the knee space. After surgery, the surgical wound was dressed without arthrodesis. Anti-infective treatment was given for 5-7 days and threads were removed at day 12 post-surgery. Most patients ambulated at 1 week post surgery and the function of knee joints was restored after 4 weeks.

Clinical assessment. Peripheral blood from all patients was collected from a cubital vein prior to treatment and at 4 days, 2 and 4 weeks after treatment. Synovial liquid was collected at the same time points. Briefly, the patients were in a supine position with limb flexion of 70-90 degrees. Following the administration of local anesthesia with $2 \%$ lidocaine, $0.5-1 \mathrm{ml}$ of synovial fluid was collected from the joint cavity. The supernatant was isolated by centrifugation at $800 \mathrm{x} \mathrm{g}$ for $10 \mathrm{~min}$ at room temperature. The serum levels of hypersensitive $C$-reactive protein (hs-CRP), the erythrocyte sedimentation rate (ESR) and synovial inflammatory factors [interleukin (IL)-1 and IL-6] were detected by immunoturbidimetry (BodiTech Med Inc., Gang-won-do, South Korea), the Westergren method (18) and ELISA [IL-1 ELISA kit; cat. no. 70-EK101B2; Hangzhou
MultiSciences (Lianke) Biotech, Co., Ltd., Hangzhou, China; IL-6 ELISA kit; cat. no. KB2730; Ke Min Biological Technology Co., Ltd., Shanghai, China].

Cure rate analysis. All patients were followed up for 2 years after the start of the treatment, and the cure rate was estimated according to a study by Lequesne et al (19) based on the severity index of osteoarthritis (including the degree of joint swelling, the grade of floating patella, joint pain and the degree of joint dysfunction). Joint swelling was graded as mild (faded skin texture and evident bone markers), moderate (obvious swelling and faint bone markers, without skin texture) and severe (significant swelling with tight skin, without bone markers). The floating patella test was evaluated by rating joints as class I (negative), II (slightly elevated patella), III (obviously elevated patella) and IV (disability of pushing the femoral condyle of the patella into the normal position). Finally, joint pain was scored as follows: Patients received one score each for pain whilst walking, pain using stairs, night pain, sitting pain and standing pain, which were added up. In the present study, KOA was considered cured if a patient presented with mild joint swelling, evident bone markers, negative floating patella test and no knee pain.

Statistical analysis. All data were analyzed using SPSS 13.0 (SPSS, Inc., Chicago, IL, USA). Normality and homogeneity of variance tests were performed for each set of data. For the data with a normal distribution and homogeneity of variance, one-way analysis of variance was used to assess the differences between the groups, and a Q test was performed for pairwise comparison among different time-points within the same group; otherwise, for data with a non-normal distribution, Dunnett's T3 multiple comparisons test was performed. $\mathrm{P}<0.05$ was considered to indicate a statistically significant difference.

\section{Results}

Demographic and clinical characteristics of KOA. A total of 23 patients with KOA (16 males and 7 females; age range, 25-45 years; 11 left and 12 right knees) were enrolled in the present study. All patients had a history of trauma with the clinical manifestations of varying degrees of joint pain or locking. The patients were previously healthy without underlying diseases of the coagulation, respiratory or circulatory system.

Plasma levels of hs-CRP in KOA patients. The plasma levels of hs-CRP and the ESR were measured to assess the disease activity in the two groups. The results demonstrated that prior to treatment, there was no significant difference in the plasma levels of hs-CRP between the conservative group and surgery group $(\mathrm{P}>0.05)$. After treatment, plasma hs-CRP in the conservative group steadily decreased in a time-dependent manner $(\mathrm{P}<0.05)$. However, the post-operative levels of plasma hs-CRP in the surgery group reached the highest value at day $4(\mathrm{P}<0.05$ vs. the conservative group). Subsequently, plasma hs-CRP levels in the surgery group decreased and were significantly lower than those in the conservative group at 2 and 4 weeks after treatment $(\mathrm{P}<0.05$; Fig. 1). 


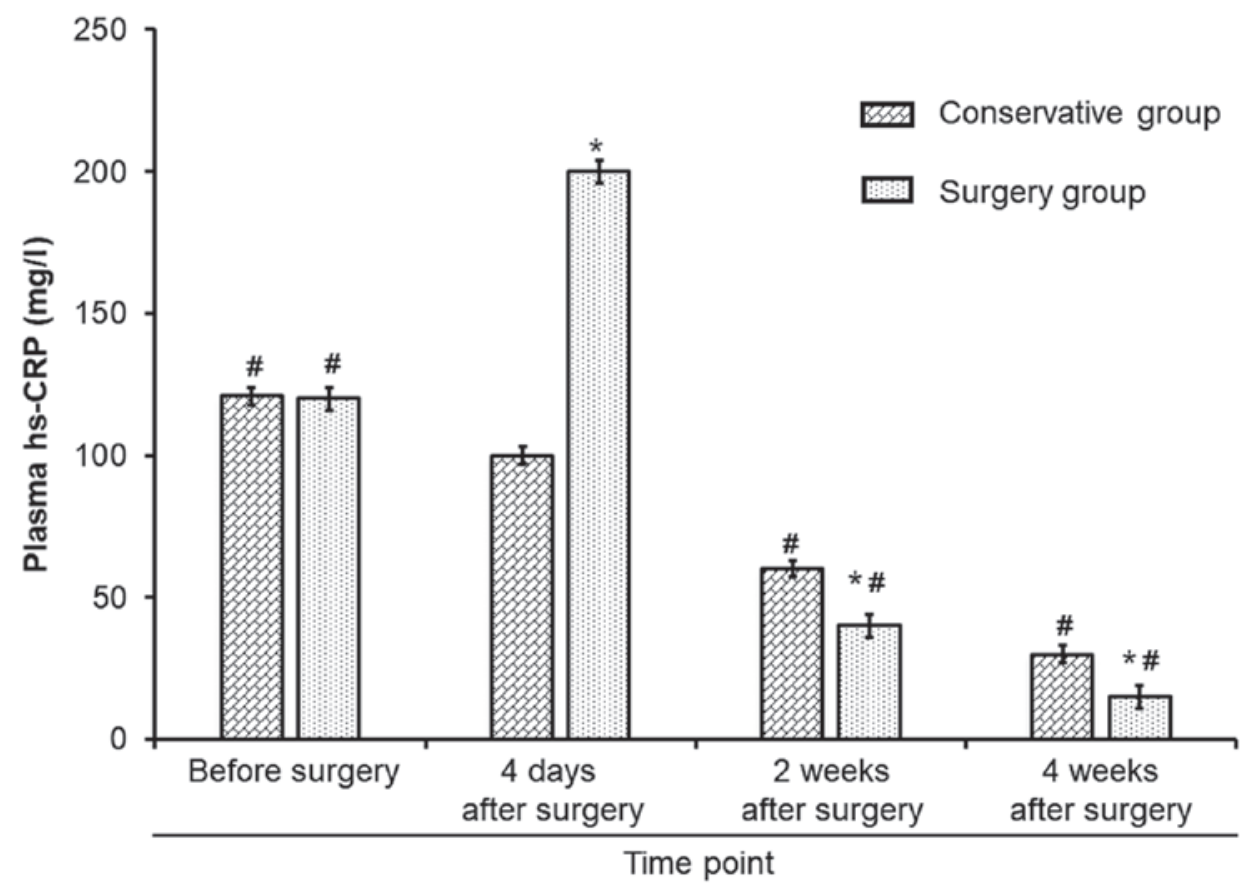

Figure 1. Plasma levels of hs-CRP. ${ }^{\mathrm{P}}<0.05$ vs. the conservative group; ${ }^{\#} \mathrm{P}<0.05$ vs. 4 days after surgery. hs-CRP, high-sensitivity $\mathrm{C}$-reactive protein.

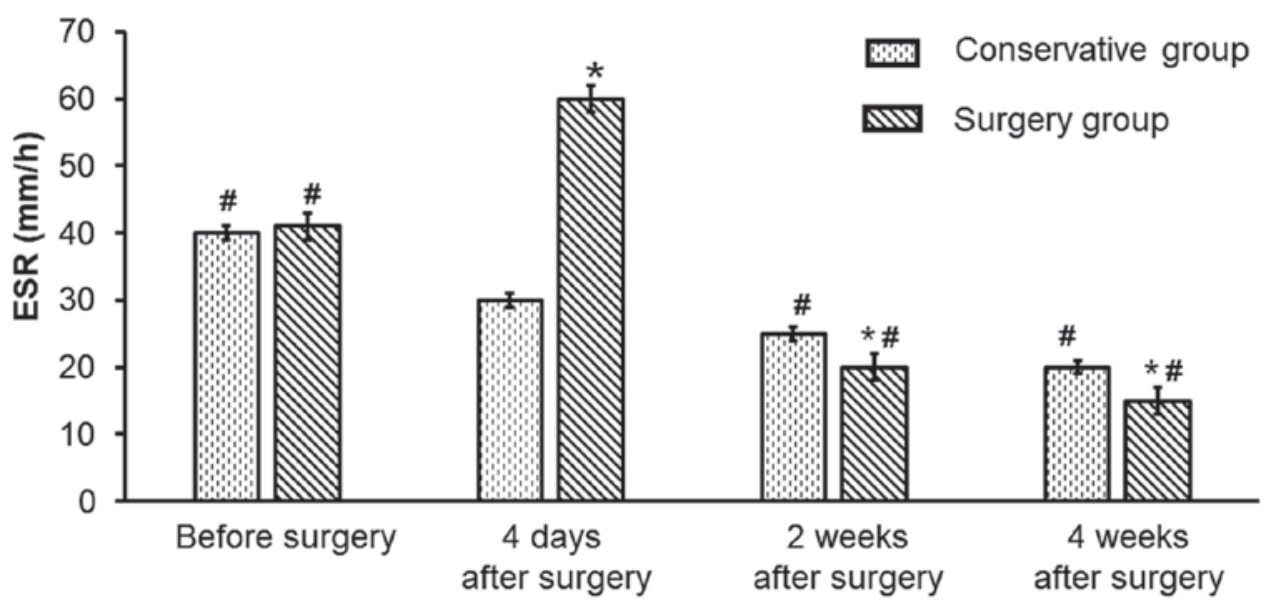

Figure 2. ESR analysis in the conservative and surgery groups. ${ }^{~} \mathrm{P}<0.05$ vs. the conservative group; ${ }^{\text {" }} \mathrm{P}<0.05$ vs. 4 days after surgery. ESR, erythrocyte sedimentation rate.

A similar trend was observed for the ESR in the conservative group and surgery group. Prior to treatment, no significant difference in the ESR was seen between the conservative group and surgery group $(\mathrm{P}>0.05)$. At 2 and 4 weeks, the post-operative ESR in the surgery group was lower than the ESR in the conservative group post-treatment (all $\mathrm{P}<0.05$ ), while at 4 days after surgery, the ESR reached a maximum value in the surgery group, which was significantly higher than that in the conservative group at 4 days after treatment $(\mathrm{P}<0.05 ;$ Fig. 2$)$. The results demonstrated that arthroscopic loose body removal may be an effective treatment of KOA.

Synovial concentration of $I L-1$ and $I L-6$ in KOA patients. Next, the levels of synovial inflammatory factors IL-1 and IL-6 were detected to evaluate the local inflammatory reaction in the joints in the two groups. Prior to treatment, no significant differences in synovial IL-1 were observed between the conservative group and surgery group $(\mathrm{P}>0.05)$. At 4 days after surgery, the IL-1 concentration in the conservative group and surgery group was increased to reach a maximum value $(\mathrm{P}<0.05)$. Subsequently, the IL-1 levels decreased in each group and remained significantly lower in the surgery group than in the conservative group at 2 and 4 weeks after treatment (all $\mathrm{P}<0.05$; Fig. 3). Similarly, compared with the levels in the conservative group, the post-operative IL-6 concentration in the surgery group was significantly lower at 2 and 4 weeks (all $\mathrm{P}<0.05$ ), despite a higher peak value at 4 days (Fig. 4). Following surgery, inflammatory factors inititally demonstrated a higher increase in the surgery group, but later a greater decrease compared with those in the conservative treatment group. This indicates that surgery initially caused more inflammation, but that the efficacy of surgery at 


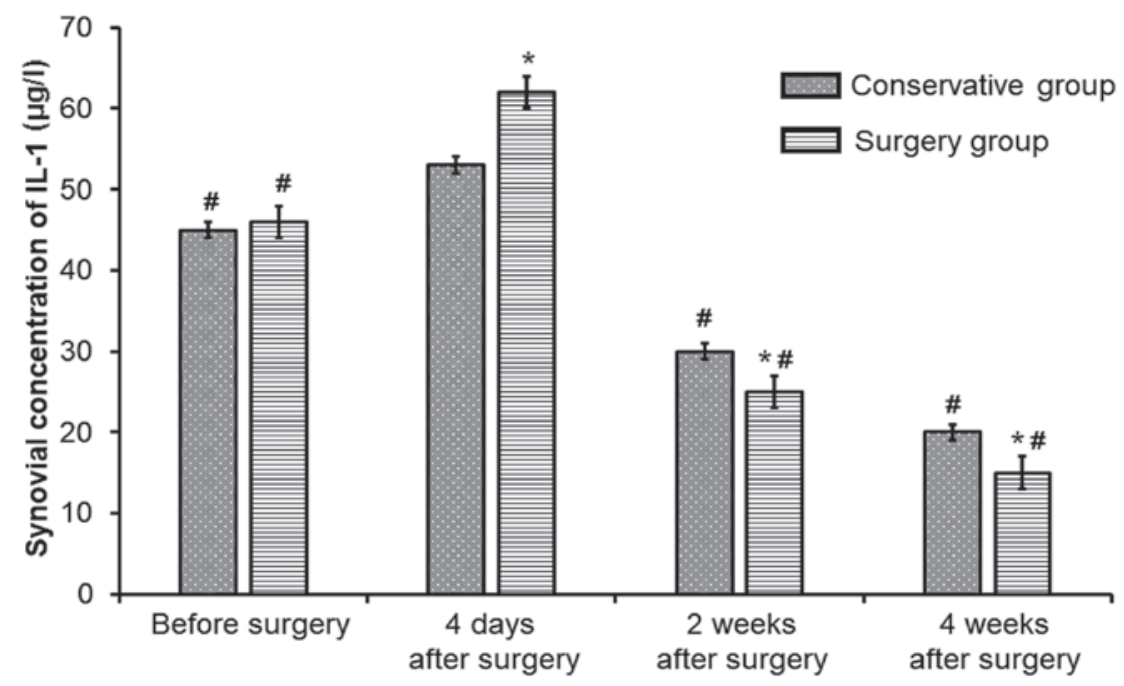

Figure 3. Synovial fluid concentration of IL-1 in the surgery and conservative groups. ${ }^{*} \mathrm{P}<0.05$ vs. the conservative group; ${ }^{\text {}} \mathrm{P}<0.05$ vs. 4 days after surgery. IL, interleukin.

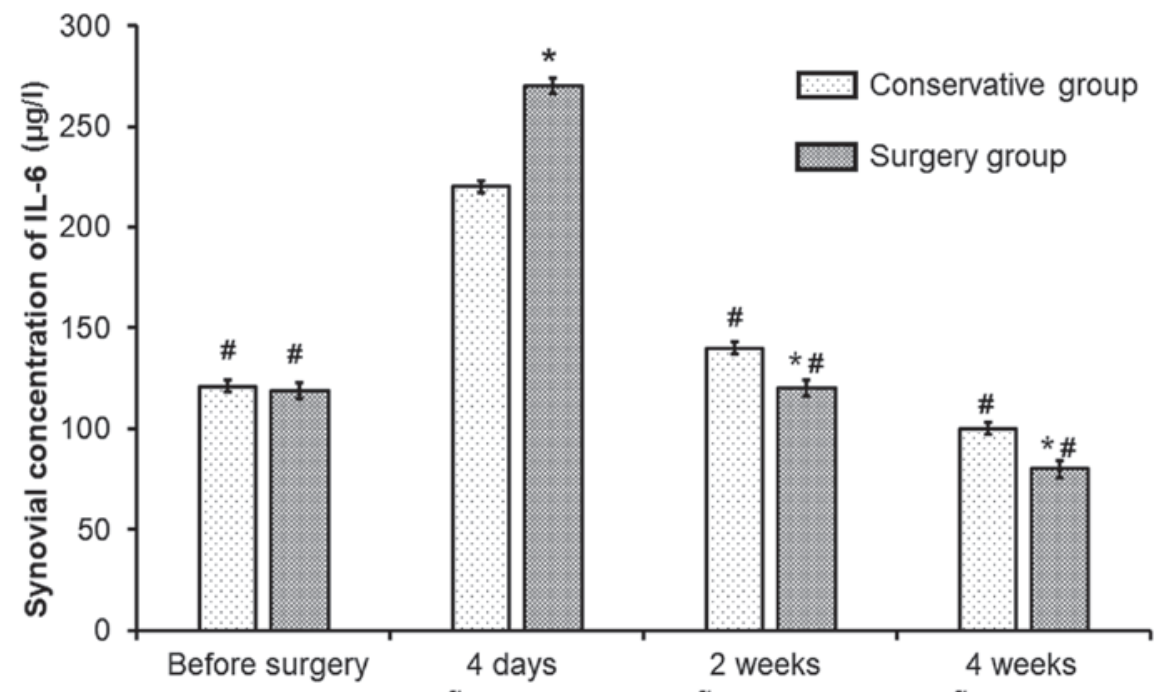

Figure 4. Synovial concentration of IL-6 in the surgery and conservative groups. ${ }^{*} \mathrm{P}<0.05$ vs. the conservative group; ${ }^{\#} \mathrm{P}<0.05$ vs. 4 days after surgery. IL, interleukin.

2 and 4 weeks was higher. These results also revealed that the local inflammatory reaction in the joints of patients with KOA is less severe after arthroscopic loose body removal than that after conservative therapy.

Comparison of cure rates for different treatments. Ultimately, the cure rate of patients in the two groups was analyzed. All patients were followed up for 2 years to estimate the cure rate. A tendency towards a higher cure rate was seen in each group with increasing follow-up time, and more importantly, the cure rate for KOA in the surgery group was significantly higher than that in the conservative group at $1,1.5$ and 2 years of follow-up $(\mathrm{P}<0.05$; Fig. 5).

\section{Discussion}

KOA is considered to be a non-inflammatory joint disease; however, inflammatory cytokines have a major role in the



Figure 5. Cure rate in the surgical and conservative groups over a 2 year period. A tendency towards a higher cure rate was observed in each group with increasing time, however, the cure rate was significantly higher in the surgery group compared with the conservative group at 1, 1.5 and 2 years of follow-up. ${ }^{*} \mathrm{P}<0.05$ vs. the conservative group. 
pathogenesis and development of osteoarthritis due to their close association with the dysfunctions of synovium, articular cartilage and subchondral bone (20). The therapeutic goals of KOA treatment are to reduce or eliminate pain, correct deformities, improve or restore joint function and improve quality of life. The main treatment approach for this disease is combination therapy including drugs, non-pharmaceutical treatments and surgical treatment if necessary.

Arthroscopy is performed for the diagnosis and treatment of joint disorders using an endoscope with diameter of $5 \mathrm{~mm}(21)$. Arthroscopic surgery is a minimally invasive surgery type and was first used in the knee joint (22). The results of the present study demonstrated that immediately after surgery, a tendency towards lower hs-CRP levels and ESR was seen in patients receiving conservative therapy. However, the post-operative levels of hs-CRP and ESR in patients with arthroscopic loose body removal reached the highest value at day 4, which may have been triggered by surgical stimulation. Subsequently, the levels of hs-CRP and ESR decreased and those in the surgery group were significantly lower than those in patients with conservative therapy at 2 and 4 weeks after surgery. Most importantly, the cure rate in patients with arthroscopic loose body removal was significantly higher than that in patients who received conservative therapy. These findings indicated that, compared with conservative treatment, arthroscopic loose body removal is more effective for KOA with a less severe inflammatory reaction once the patients recovered from surgery.

Previous studies have indicated that IL-1 and IL-6 have critical roles in the pathological process of osteoarthritis via promoting cartilage matrix degradation and damaging articular cartilage $(23,24)$. It has been demonstrated that IL-1 and IL-6 stimulate a variety of mesenchymal cells to release proteolytic enzymes, co-stimulate antigen-presenting cells and activate $\mathrm{T}$ cells, promote $\mathrm{B}$ cell proliferation as well as antibody secretion and subsequently regulate the metabolism of cartilage cells, fibroblasts and bone cells (25-27). Synovial lesions in OA are due to decomposition of synovial tissue by prostaglandins and collagenase secreted by stromal cells after IL-1 stimulation in joint capsules (28). Previous studies revealed that the expression levels of IL-1 $\beta$ in the synovial fluid of patients with KOA was increased and positively correlated with articular cartilage damage $(29,30)$. IL-1 also induces the degeneration of cartilage and inhibition of chondrocyte proliferation though disturbing the mechanism of collagen I-IV in patients with KOA (31). In the present study, the synovial fluid levels of IL-1 and IL-6 in patients immediately after arthroscopic loose body removal and conservative therapy were higher than those prior to treatment, which was consistent with the findings of Marks and Donaldson (29). Furthermore, at 4 days after surgery, the IL-1 and IL-6 concentration in patients with arthroscopic loose body removal and those with conservative therapy was increased to reach a maximum value. Subsequently, the levels of these inflammatory cytokines decreased and remained lower in patients with arthroscopic loose body removal than that in patients with conservative therapy at 2 and 4 weeks after surgery. One reasonable speculation regarding the transient peak value of IL- 1 and IL- 6 is that it may be induced by surgical stress.
In conclusion, arthroscopic loose body removal for treating KOA, which also has the advantage of minimal surgical incision, less scarring and fewer complications (32), is a more effective treatment for KOA.

\section{Acknowledgements}

The authors wish to thank Professor Xin Liu (Department of Orthopedics, Linyi People's Hospital, Linyi, China) for his technical support.

\section{References}

1. Woolf AD and Pfleger B: Burden of major musculoskeletal conditions. Bull World Health Organ 81: 646-656, 2003.

2. Murray CJ, Vos T, Lozano R, Naghavi M, Flaxman AD, Michaud C, Ezzati M, Shibuya K, Salomon JA, Abdalla S, et al: Disability-adjusted life years (DALYs) for 291 disease and injuries in 21 regions, 1990-2010: A systematic analysis for the Global Burden of Disease Study 2010. Lancet 380: 2197-2223, 2012.

3. Vos T, Flaxman AD, Naghavi M, Lozano R, Michaud C, Ezzati M, Shibuya K, Salomon JA, Abdalla S, Aboyans V, et al: Years lived with disability (YLDs) for 1160 sequelae of 289 diseases and injuries 1990-2010: A systematic analysis for the Global Burden of Disease Study 2010. Lancet 380: 2163-2196, 2012.

4. Nishimura A, Hasegawa M, Wakabayashi H, Yoshida K, Kato K, Yamada T, Uchida A and Sudo A: Prevalence and characteristics of unilateral knee osteoarthritis in a community sample of elderly Japanese: Do fractures around the knee affect the pathogenesis of unilateral knee osteoarthritis? J Orthop Sci 17: 556-561, 2012.

5. Wang XQ: Osteoarthritis: Diagnosis and treatment. Tianjin Science and Technology Translation and Publishing Corporation, Tianjin, p292, 2005.

6. Deng ZQ, Zhang RL and Yu D: Chinese medicine combined with physical manipulation on knee osteoarthritis: A report of 106 cases. Chin J Misdiagnosis 8: 8466, 2008 (In Chinese).

7. Turajane T, Tanavaree A, Labpiboonpong V and Maungsiri S: Outcomes of intra-articular injection of sodium hyalumnate for the treatment of osteoarthris of the knee. J Med Assoc Thai 90: 1845-1852, 2007.

8. Liu KM and Liu XL: Progress of rehabilitation and therapy for knee osteoarthritis. Chin J Clinicians 6: 972-976, 2012 (In Chinese).

9. Manheimer E, Linde K, Lao L, Bouter LM and Berman BM: Meta-analysis: Acupuncture for osteoarthritis of the knee. Ann Intern Med 146: 868-877, 2007.

10. Hu S and Li Y: Progress in pathological changes of osteoarthritis. Pract J Clin Med 8: 87-88, 2011 (In Chinese).

11. Schnizer TJ: Update on guidelines for the treatment of chronic musculoskeletal pain. Clin Rheumatol 25 (Suppl 1): S22-S29, 2006.

12. Lützner J, Kasten P, Günther KP and Kirschner S: Surgical options for patients with osteoarthritis of the knee. Nat Rev Rheumatol 5: 309-316, 2009.

13. Milgram JW: The classification of loose bodies in human joints Clin Orthop Relat Res: 282-291, 1977.

14. Kim HG, Park KH, Huh JK, Song YB and Choi HS: Magnetic resonance imaging characteristics of synovial chondrimatisis of the temporomandibular joint. J Orofac Pain 16: 148-153, 2002.

15. Xu B and Xu HG: Morphological and physiological features of cartilaginous loose bodies in the knee joint. J Clin Rehabilitative Tissue Eng Res 11: 1009-1013, 2007.

16. Li XS, Zhou JN and Lei GH: Experience of 21 Cases of Excision Corpus Liberum of Knee Joint by Arthroscopy. China J Endoscopy 8: 62-63, 2002 (In Chinese).

17. Sun PH: Loose body removal surgery for Beck disease: Clinical Summary of 404 cases. J Pract Med Tech 15: 3385-3386, 2008 (In Chinese).

18. ICSH recommendations for measurement of erythrocyte sedimentation rate. International Council for Standardization in Haematology (Expert Panel on Blood Rheology). J Clin Pathol 46: 198-203, 1993.

19. Lequesne MG, Mery C, Samson M and Gerard P: Indexes of severity for osteoarthritis of the hip and knee. Validation-value in comparison with other assessment tests. Scand J Rheumatol Suppl 65: 85-89, 1987. 
20. Arthroscopic knee surgery therapy, http://www.nohl8.com/gujye/ gujejin.htm, 2010. 02.27.

21. Yang XM, Liu YL and Ren CL: Operative cooperation of arthroscopy for knee surgery. J Pract Med Tech 12: 1748-1749, 2005 (In Chinese).

22. Gibson JN, White MD, Chapman VM and Strachan RK: Arthroscopic lavage and debridement for osteoarthritis of the knee. J Bone Joint Surg Br 74: 534-537, 1992.

23. Pelletier JP, Martel-Pelletier J and Abramson SB: Osteoarthritis, an inflammatory disease: Potential implication for the selection of new therapeutic targets. Arthritis Rheum 44: 1237-1247, 2011

24. Wang HJ, Yu CL, Kishi H, Motoki K, Mao ZB and Muraguchi A: Suppression of experimental osteoarthritis by adenovirus-mediated double gene transfer. Chin Med J (Engl) 119: 1365-1373, 2006.

25. Lianxu C, Hongti J and Changlong Y: NF-kappaBp65-specific siRNA inhibits expression of genes of COX-2, NOS-2 and MMP-9 in rat IL-1beta-induced and TNF-alpha-induced chondrocytes. Osteoarthritis Cartilage 14: 367-376, 2006.

26. Xiang C, Liu J, Wei XC and Geng JH: In vitro transfection of interleukin-1Ra and transforming growth factor- $\beta 1$ mediated with retrovirus PLNCX2 into knee articular chondrocytes of rabbits. Chin J Experimental Surg 30: 247-251, 2013 (In Chinese).
27. Steinbrech DS, Mehrara BJ, Rowe NM, Dudziak ME, Luchs JS, Saadeh PB, Gittes GK and Longaker MT: Gene expression of TGF-beta, TGF-beta reporter, and extracellular matrix proteins during membranous bone healing in rats. Plast Reeonstr Surg 105: 2028-2038, 2000

28. Tischer T, Aktas T, Milz S and Putz RV: Detailed pathological changes of human lumbar facet joints L1-5 in elderly individuals. Eur Spine J 15: 308-315, 2006.

29. Marks PH and Donaldson ML: Inflammatory cytokine profiles associated with chondral damage in the anterior cruciate ligament-deficient knee. Athroscopy 21: 1342-1347, 2005.

30. Aigner T, McKenna L, Zien A, Fan Z, Gebhard PM and Zimmer R: Gene expression profiling of serum- and interleukin-1beta stimulated primary human adult articular chondrocytes-A molecular analysis based on chondrocytes isolated from one donor. Cytokine 31: 227-240, 2005.

31. Chen WJ: The research progress of Interleukin-1a family and osteoarthritis. Chongqing Med J 40: 3198-3200, 2011 (In Chinese)

32. Homandberg GA, Umadi V and Kang H: High molecular weight hyaluronan promotes repair of IL-1 beta-damaged cartilage explants form both young and old bovines. Osteoarthritis Cartilage 11: 177-186, 2003. 